ISSN 0103-9954

\title{
ESTRUTURA E PADRÕES DE DISTRIBUIÇÃO ESPACIAL DE ESPÉCIES ARBÓREAS EM UMA AMOSTRA DE FLORESTA OMBRÓFILA MISTA EM NOVA PRATA, RS
}

\section{STRUCTURE AND SPATIAL DISTRIBUTION PATTERNS OF TREE SPECIES IN A MIXED OMBROPHYLOUS FOREST SAMPLE IN NOVA PRATA, RS}

\author{
André R. Terra Nascimento ${ }^{1}$ Solon Jonas Longhi ${ }^{2}$ Doádi Antônio Brena ${ }^{3}$
}

\section{RESUMO}

Este estudo objetiva descrever a estrutura da vegetação e os padrões de distribuição espacial das espécies arbóreas e arvoretas de uma amostra de um hectare de Floresta Ombrófila Mista, localizada no município de Nova Prata, estado do Rio Grande do Sul. Utilizando o método de área fixa, foram instaladas vinte unidades amostrais de 10 x $50 \mathrm{~m}\left(500 \mathrm{~m}^{2}\right)$, perfazendo uma amostra de $100 \times 100 \mathrm{~m}\left(10000 \mathrm{~m}^{2}\right)$ e mensurados todos os indivíduos com DAP $\geq 9,6 \mathrm{~cm}(30 \mathrm{~cm}$ de circunferência), que compõem o dossel da floresta. A amostra de vegetação estudada apresentou uma diversidade mediana de espécies arbóreas e arvoretas (55 espécies com DAP $\geq 9,6 \mathrm{~cm}$ ) e uma elevada densidade de indivíduos por hectare (848 ind./hectare), similares aos encontrados em florestas secundárias, no domínio da Floresta Ombrófila Mista. A vegetação, na amostra, apresentou-se com maior proporção de espécies com distribuição agregada ou com tendência à agregação $(46,7 \%)$, revelando que as maiores populações arbóreas formam pequenas manchas ou agrupamentos na vegetação, com maiores valores obtidos para a Razão Variância/Média. Este estudo, mesmo se tratando de uma análise em pequena escala espacial $\left(10000 \mathrm{~m}^{2}\right)$, destaca a densidade e a distribuição das espécies arbóreas a arbustivas em um trecho de floresta que sofreu exploração comercial, fragmentos importantes das florestas atuais do estado do Rio Grande do Sul, Brasil.

Palavras-chave: distribuição espacial, Floresta Ombrófila Mista, fitossociologia.

\begin{abstract}
The objective of this study is to describe the vegetation structure and the spatial distribution patterns of tree and shrub species in one hectare of a Mixed Ombrophylous Forest, localized in the County of Nova Prata, state of Rio Grande do Sul, Brazil. Using the fixed area method, were installed 20 samples of $10 \times 50 \mathrm{~m}\left(500 \mathrm{~m}^{2}\right)$, completing one sample of the $100 \times 100 \mathrm{~m}\left(10000 \mathrm{~m}^{2}\right)$ and measured all individuals with diameter at breast height $(\mathrm{DBH}) \geq 9,6 \mathrm{~cm}$ (30 cm of circumference), that make up the canopy of the forest. The sample of vegetation studied showed a

1. Engenheiro Florestal, MSc., Embrapa Recursos Genéticos e Biotecnologia, Laboratório de Ecologia de Plantas, Parque e Estação Biológica, CEP 70770-900, Brasília (DF). andre@ cenargen.embrapa.br

2. Engenheiro Florestal, Dr., Professor Titular do Departamento de Ciências Florestais, Centro de Ciências Rurais, Universidade Federal de Santa Maria, CEP 97105-900, Santa Maria (RS). longhiso@ccr.ufsm.br

3. Engenheiro Florestal, Dr., Professor Adjunto do Departamento de Ciências Florestais, Centro de Ciências Rurais, Universidade Federal de Santa Maria, CEP 97105-900, Santa Maria (RS).brena@ccr.ufsm.br
\end{abstract}


medium diversity of arboreal and shrub species (55 species with $\mathrm{DBH} \geq 9,6 \mathrm{~cm}$ ) and a large density of individuals per ha (848 individuals/ha), similar to the ones found in secondary forests, in the domain of the Mixed Ombrophylous Forest. The vegetation in the sample presented a larger proportion of species aggregated or with aggregation tendency $(46,7 \%)$, revealing that the larger tree population make up small spots or groups in the vegetation, with larger values obtained for the Variance/Average rate. This study, even though treating of a small spatial scale analyze $\left(10000 \mathrm{~m}^{2}\right)$, depict the density and the distribution of the arboreal and shrubs species in a part of secondary forest, that was damaged by commercial exploitation important fragment of the present forests of the state of Rio Grande do Sul, Brazil.

Key words: spatial distribution, Mixed Ombrophylous Forest, phytossociology.

\section{INTRODUÇÃO}

O Brasil é um país detentor de uma grande diversidade biológica, encontrada nos diversos ecossistemas existentes com suas numerosas formas de vida. O conhecimento sobre a dinâmica dessas comunidades é de grande importância para sua conservação, pois esses remanescentes contem populações de animais e plantas que, atualmente, se tornaram raros ou em vias de extinção. A utilização dos recursos florestais pelo homem, através dos tempos, provocou uma redução drástica das diversas comunidades vegetais com características bastante peculiares, comprometendo a sustentabilidade e a posterior manutenção da diversidade biológica destas.

A Floresta Ombrófila Mista (Floresta com Araucária) possui remanescentes florestais nos estados do Rio Grande do Sul, Santa Catarina, Paraná, São Paulo e Minas Gerais (INOUE et al., 1984). Atualmente, encontra-se bastante fragmentada, com escassos remanescentes que representem uma amostra adequada desse tipo de vegetação para a sua conservação a longo prazo.

A exploração intensiva de madeiras de grande valor econômico de espécies como Araucaria angustifolia (pinheiro-brasileiro), Ocotea porosa (imbuia), Luehea divaricata (açoita-cavalo) e Cedrela fissilis (cedro) reduziu suas reservas naturais, o que, aliado à falta de estudos sobre a demografia e a dinâmica na comunidade, colocam essas populações residuais em grande perigo. Outras espécies não-madeiráveis, comuns nessa formação vegetal, como Dicksonia sellowiana (xaxim) e Maytenus ilicifolia (espinheira-santa), exploradas como produtos secundários, sofreram uma grande redução em suas reservas naturais.

Estudos sobre a composição e a estrutura desses remanescentes florestais fornecem informações básicas para tomadas de decisões na aplicação de técnicas de manejo florestal ou conservação destes. A estrutura da Floresta Ombrófila Mista é complexa e os conhecimentos sobre os diversos tipos de comunidades, que existem dentro de sua área de distribuição natural, ainda não permitem uma política de conservação eficiente que mantenha a maior parte de sua diversidade vegetal pouco conhecida.

Em uma comunidade vegetal, os seus constituintes (plantas) encontram-se arranjados conforme as diversas associações naturais, que uma determinada vegetação possui, ao longo de sua 
distribuição natural. Dessa forma, segundo DAJOZ (1972), podem ser reconhecidos basicamente os seguintes tipos de distribuição espacial: uniforme (regular), ao acaso (aleatório) e contagiosa (agregada).

O presente estudo objetiva analisar a estrutura fitossociológica da vegetação e também estimar os diferentes padrões de distribuição espacial de indivíduos adultos, em uma amostra de um hectare de floresta em estado de recuperação, após exploração seletiva de seus indivíduos comerciais, dentro do domínio da Floresta Ombrófila Mista no estado do Rio Grande do Sul.

\section{REVISÃO DE LITERATURA}

\section{Conceitos sobre a composição e a estrutura da floresta Ombrófila Mista}

Na Floresta Ombrófila Mista, a Araucaria angustifolia, que constitui o dossel superior dessa tipologia florestal, é uma espécie que apresenta um caráter dominante na vegetação, representando uma grande porcentagem dos indivíduos do estrato superior e ocorrendo como espécie emergente, atingindo grandes alturas e diâmetros (LONGHI, 1980; LEITE \& KLEIN, 1990). Essa conífera, sem dúvida, é a espécie mais importante que caracteriza a fitofisionomia dessa formação florestal.

Com relação à estrutura, as florestas com Araucaria apresentam forma bastante flexível, podendo ser uni ou multiestratificada e variar de coetânea a multietânea (SCHIMIDT et al., 1980). O pinheiro-brasileiro apresenta uma grande contribuição na estrutura da floresta, dominando o estrato superior e permanecendo como indivíduos senis nas florestas mais desenvolvidas (LONGHI, 1980; JARENKOW, 1985).

Em uma Floresta Ombrófila Mista em Aracuri, Esmeralda- RS, JARENKOW \& BATISTA (1987) destacaram que a estratificação não se encontrava muito definida. Esses autores citam a existência de três estratos arbóreos descontínuos, o primeiro dominado pelo pinheiro-brasileiro com alturas de até $28 \mathrm{~m}$, um segundo estrato entre 12 e $19 \mathrm{~m}$ de altura, e um terceiro estrato com alturas distribuídas entre $5 \mathrm{~m}$ e $12 \mathrm{~m}$.

A ocorrência de espécies epífitas, das famílias Bromeliaceae e Orchidaceae é pouco acentuada na estrutura da vegetação estudada. A maior participação, nesse componente da vegetação, são de espécies vegetais das famílias Aspleniaceae, Polypodiaceae e Hymenophyllaceae como descrevem SENNA \& WAECHTER (1997), que recobrem os troncos das árvores, dando o aspecto que RAMBO (1956) denominou de "Matas pretas".

Embora Araucaria angustifolia apresente predominância no estrato superior da floresta, os estratos médio e inferior possuem grande diversidade de espécies que se regeneram nesse ambiente. Esses estratos são importantes centros de dispersão de um grande número de espécies, principalmente, das pertencentes às famílias Myrtaceae e Lauraceae, como descrevem RAMBO (1951) e KLEIN (1984).

LONGHI (1997), estudando o agrupamento de comunidades vegetais da Floresta Ombrófila Mista na bacia do rio Passo Fundo/RS, mediante técnicas estatísticas multivariadas, encontrou seis 
diferentes agrupamentos na vegetação. Cada agrupamento apresentou uma composição de espécies vegetais características, identificando diferentes unidades na vegetação (associações vegetais) dentro da área estudada. As comunidades mais avançadas, em termos sucessionais, estudadas pelo autor, foram as associações naturais denominadas de Canela-preta (Nectandra megapotamica) e Araucária (Araucaria angustifolia), referindo-se à elevada densidade dessas duas espécies em cada uma das associações.

As florestas de Gimnospermas são em geral gregárias, compondo florestas relativamente homogêneas. Mesmo em países tropicais como o Brasil, pode ser observada essa tendência gregária. Embora associados a uma diversificada flora angiospérmica, os pinhais de Araucaria angustifolia também se distinguem pelo elevado número de indivíduos dessa espécie por unidade de área (MARCHIORI, 1996).

Essa formação florestal, apresenta estrutura extremamente variável, ora apresentando agrupamentos densos com abundância de Lauraceae, ora apresentando agrupamentos pouco desenvolvidos com um predomínio de Podocarpus, Drymis e Aquifoliaceae (LEITE \& KLEIN, 1990). Na região de Nova Prata, a Floresta Ombrófila Mista apresenta uma estrutura complexa e pluri-estratificada, onde destacam-se, nos primeiros estratos da vegetação, as famílias Myrtaceae, Sapindaceae e Lauraceae em relação ao número de espécies de árvores e arvoretas, onde predominam Matayba elaeagnoides (camboatá-branco), Zanthoxylum kleinii (juvevê), Araucaria angustifolia (pinheiro-brasileiro), Blepharocalyx salicifolius (murta), Cupania vernalis (camboatávermelho), entre outras (NASCIMENTO, 2000).

\section{Padrões de distribuição espacial e alguns Índices de dispersão}

O padrão de distribuição espacial de uma espécie é representado pela sua distribuição na área em estudo, em termos de freqüência de ocorrência dentro das unidades amostrais coletadas (JANKAUSKIS, 1990).

Uma espécie vegetal, embora apresente uma grande ocorrência em uma determinada área, sua distribuição espacial, nas diferentes classes de tamanho, pode ser bastante irregular. O grau de agregação pode apresentar diferentes valores, com as plantas das menores classes de tamanho apresentando tendência ao agrupamento e as plantas das maiores classes de tamanho podendo ocorrer de maneira fortemente agrupadas (CARVALHO, 1983).

Em estudos com unidades de amostra (quadras), o índice de agregação de Morisita é pouco influenciado pelo tamanho da unidade amostral, apresentando excelentes qualidades de detecção (Brower \& Zar citados por BARROS, 1986). O teste de ajuste da distribuição de Poisson mediante o Qui-quadrado, juntamente com o índice de agregação de McGUINNES, permitem também detectar a presença de agregação em espécies vegetais (BARROS, 1986; CARVALHO, 1992).

Entre os métodos mais utilizados para o estudo da distribuição espacial de indivíduos adultos, destacam-se principalmente o método dos quadrados - que emprega distribuições generalizadas como a Binomial e Poisson; e o método das distâncias - que usa distâncias das plantas selecionadas para outras plantas ou pontos aleatórios para plantas adjacentes (SILVA \& LOPES, 1982).

Para as espécies vegetais, que apresentam uma distribuição com tendência à agregação, pode, 
muitas vezes, ser difícil de descrever esse agrupamento na vegetação. McGHINES (1934) criou um índice que relaciona a Abundância Relativa e a Freqüência Absoluta, possibilitando uma melhor análise e compreensão da distribuição espacial de uma Floresta Seca da América do Norte.

Posteriormente, usando esses mesmos parâmetros estatísticos para a análise da distribuição espacial das espécie arbóreas de uma Floresta de Terra Firme na Amazônia, JARDIM (1989; 1990) propôs um índice de Dispersão (ID) que relaciona a Frequiência absoluta (FA) e a Abundância relativa $(\mathrm{AB} \%)$, atualmente designada de Densidade Relativa (DR), possibilitando a descrição dos diferentes padrões de distribuição espacial de indivíduos adultos na comunidade.

Comparando sete diferentes índices de distribuição espacial de indivíduos adultos de espécies arbóreas, CONDES \& MARTINEZ-MILLAN (1998) concluem que os índices de Clark \& Evans e Byth \& Ripley são os mais eficientes para detectar os padrões na vegetação estudada. O primeiro índice requer o conhecimento do número de indivíduos por hectare o que se supõe ser uma desvantagem em relação ao segundo índice. As diferenças, em relação aos índices restantes, são menos significativas, e o índice de Hopkins \& Skellam é menos eficiente, no caso de discriminar entre distribuições aleatórias e distribuições agregadas.

Espécies vegetais, que pertencem aos estágios iniciais de sucessão e que habitam locais alterados como grandes clareiras e bordas de vegetação, parecem apresentar um padrão de distribuição gregário. Essas espécies possuem um caráter agressivo, adaptadas às variadas condições ecológicas, e apresentando geralmente uma arquitetura simples (NASI, 1993).

Nesse sentido, as plantas com frutos zoocóricos, podem apresentar uma distribuição das sementes em forma de montes "clump-dispersal" ou na forma aleatória "scatter-dispersal", descritas por HOWE (1989) e também discutidos por HOWE e WESTLEY (1988). As sementes, principalmente das espécies com frutos grandes, geralmente caem embaixo da copa da árvore e, com isso, podem ser predadas por insetos e roedores e, em alguns casos, germinarem em alta competição, no processo de seleção natural dessas populações em regeneração natural. Nos períodos iniciais de estabelecimento dos novos indivíduos, as taxas de predação de sementes podem ser bastante elevadas.

Comparando a estrutura e a dinâmica de duas florestas de galeria, BELEM (1997) descreve alguns padrões de distribuição espacial dos indivíduos adultos em amostras quadradas de um hectare. Grandes árvores, como Ceiba pentandra e Nauclea lancifolia, apresentaram uma distribuição aleatória na área e também baixos valores de densidade, demonstrando diferenças estruturais e espaciais nas duas comunidades em relação às espécies com maior densidade e que se regeneram em cada um dos sítios estudados pelo autor.

Os efeitos da heterogeneidade espacial, no tamanho da estrutura populacional, são análogos aos efeitos da heterogeneidade espacial em diversidade de espécies. Em nível de comunidade e para organismos similares, mas especialmente separados, que, provavelmente, não competem um com o outro pelas condições ambientais, apresentam alto padrão de heterogeneidade espacial capaz de, potencialmente, permitir a coexistência de muitas espécies diferentes e, dessa forma, uma alta diversidade de espécies desses locais (HUSTON, 1994). 


\section{MATERIAL E MÉTODOS}

\section{Localização da área de estudo}

Este estudo foi conduzido em um remanescente de Floresta Ombrófila Mista, localizado no município de Nova Prata, região do planalto das Araucárias do estado do Rio Grande do Sul. A área, conhecida por Fazenda Tupi, localizada entre as coordenadas $28^{\circ} 56^{\prime}$ de latitude sul e $51^{\circ} 53^{\prime}$ de longitude oeste de Greenwich, possui cerca de 720 hectares cobertos por floresta de pinheiro, estando constituído de um mosaico de trechos em diferentes estádios de sucessão, cerca de 20 anos após esta ter sofrido intensa exploração seletiva de indivíduos comerciais, e áreas com atividades agropecuárias.

\section{Método de análise}

Os indivíduos adultos foram mensurados em uma amostra de 100 x $100 \mathrm{~m}\left(10000 \mathrm{~m}^{2}\right)$ dividida em vinte unidades amostras de $10 \mathrm{~m}$ x $50 \mathrm{~m}\left(500 \mathrm{~m}^{2}\right)$, instaladas para o estudo de dinâmica florestal (BRENA et al., 1990). Para cada indivíduo amostrado, foi mensurado o CAP (circunferência à altura do peito $\geq 30 \mathrm{~cm}$ ), altura total, altura comercial e posição em relação ao dossel e anotada a posição de cada planta na parcela (coordenadas). No centro da parcela, em uma faixa de $10 \mathrm{~m}$ x $50 \mathrm{~m}\left(500 \mathrm{~m}^{2}\right)$, foi representado o perfil longitudinal da vegetação, tomando por base a altura vertical e a posição da espécie na amostra, buscando complementar o conhecimento dos aspectos da estrutura do dossel da floresta e representar as principais eco-unidades encontradas na vegetação (NASCIMENTO, 2000).

Para a identificação das espécies foram feitas coletas de material botânico. As exsicatas desse material encontram-se incorporadas no Herbário do Departamento de Ciências Florestais (HDCF) da UFSM. Para algumas espécies, principalmente da família Myrtaceae, que possui uma grande importância ecológica na vegetação, contou-se com a colaboração do pesquisador Marcos Sobral, da UFRGS.

Os parâmetros fitossociológicos foram calculados pelo "Software" Fitopac 2, desenvolvido pelo Professor George J. Shepherd, da UNICAMP - São Paulo. O programa calcula os parâmetros fitossociológicos tradicionais (densidade, dominância, freqüência, valor de importância, índice de diversidade de Shannon, entre outros) freqüentemente utilizados por diversos autores, entre eles CAIN et al. (1956), LAMPRECHT (1962), DAUBENMIRE (1968), FINOL (1971), MUELLLERDOMBOIS \& ELLENBERG (1974), LONGHI (1980, 1997) e MARTINS (1991).

\section{Estimativa dos padrões de distribuição espacial para as espécies selecionadas}

Mediante os valores estimados para as Médias e Variâncias do número de indivíduos nas 20 unidades de $10 \mathrm{~m}$ x $50 \mathrm{~m}\left(500 \mathrm{~m}^{2}\right)$ e, com base nos valores de Densidade e Freqüência foi calculada a Razão Variância/Média, também conhecida como índice de PAYANDEH, usado por CARVALHO (1983) e CALDATO (1998):

Segundo esse autor, o índice de agregação (P) é expresso: 
$\mathrm{P}=\mathrm{V} / \mathrm{M}$

Sendo: $\mathrm{P}$ = índice de agregação.

$\mathrm{V}=$ variância do número de plantas por quadrado.

$\mathrm{M}=$ média do número de plantas por quadrado.

Os valores de $\mathrm{P}$, menores que 1,0, indicam a inexistência de agrupamento. Valores de $\mathrm{P}$ entre 1,0 e 1,5 indicam tendência ao agrupamento, e os valores maiores que 1,5 indicam agrupamento.

$\mathrm{Na}$ análise dos parâmetros da distribuição (Densidade e Freqüência) e dos padrões de distribuição, somente foram consideradas as espécies com mais de dois indivíduos nas amostras, desconsiderando as espécies consideradas como raras, ou seja, espécies vegetais com densidade igual ou inferior a dois indivíduos por hectare.

\section{RESULTADOS E DISCUSSÃO}

\section{Diversidade florística e estrutura do dossel da floresta}

Foram amostrados, no hectare da floresta, 848 indivíduos com DAP $\geq 9,6$ centímetros, pertencentes a 54 espécies arbóreas, distribuídas em 23 famílias botânicas (Tabela 1). A vegetação analisada, corresponde a uma floresta em estádio de sucessão secundária, com um número não muito elevado de espécies arbóreas. Outro aspecto, que auxilia nessa afirmação, é que a floresta sofreu intervenções sem-corte raso, com a retirada de alguns elementos constituintes do dossel da floresta, ocasionando uma maior ocorrência de clareiras no dossel.

TABELA 1: Parâmetros fitossociológicos para as espécies arbóreas e arvoretas, amostradas com DAP $\geq 9,6 \mathrm{~cm}$, em Floresta Ombrófila Mista, Nova Prata, RS.

\begin{tabular}{|c|c|c|c|c|c|c|c|c|}
\hline FAMÍLIA & ESPÉCIES & $\mathrm{DA}$ & DR & FA & FR & DoA & DoR & V I \\
\hline Anacardiaceae & Lithraea brasiliensis L. March. & 55 & 6,49 & 80 & 4,97 & 3,3193 & 10,22 & 21,68 \\
\hline Anacardiaceae & Schinus terebenthifolius Raddi & 2 & 0,24 & 10 & 0,62 & 0,0551 & 0,17 & 1,03 \\
\hline Aquifoliaceae & Ilex brevicuspis Reissek & 9 & 1,06 & 45 & 2,80 & 0,4423 & 1,36 & 5,22 \\
\hline Aquifoliaceae & Ilex sp. & 11 & 1,30 & 40 & 2,48 & 0,5545 & 1,71 & 5,49 \\
\hline Araucariaceae & Araucaria angustifolia (Bert.)Ktze. & 23 & 2,71 & 55 & 3,42 & 4,1517 & 12,79 & 18,92 \\
\hline Arecaceae & Syagrus romanzoffiana (Cham.) Glassman & 3 & 0,35 & 15 & 0,93 & 0,1576 & 0,48 & 1,76 \\
\hline Bignociaceae & Tabebuia alba (Cham.) Sandw. & 1 & 0,12 & 5 & 0,31 & 0,0466 & 0,14 & 0,57 \\
\hline Erythroxylaceae & Erythroxylum deciduum St. Hil. & 70 & 8,25 & 90 & 5,59 & 2,026 & 6,24 & 20,08 \\
\hline Euphorbiaceae & Sapium glandulatum (Vell.) Pax. & 1 & 0,12 & 5 & 0,31 & 0,0368 & 0,11 & 0,54 \\
\hline Euphorbiaceae & Sebastiania commersoniana (Baill.) S. \& D. & 10 & 1,18 & 15 & 0,93 & 0,1565 & 0,48 & 2,59 \\
\hline Flacourtiaceae & Banara tomentosa $\mathrm{Clos}$ & 13 & 1,53 & 30 & 1,86 & 0,1561 & 0,48 & 3,87 \\
\hline Flacourtiaceae & Casearia decandra Jacq. & 8 & 0,94 & 30 & 1,86 & 0,1637 & 0,50 & 3,30 \\
\hline Flacourtiaceae & Xylosma tweedianum (Clos.) Eichl. & 1 & 0,12 & 5 & 0,31 & 0,0215 & 0,07 & 0,50 \\
\hline Lauraceae & Cryptocaria aschersoniana $\mathrm{Mez}$ & 1 & 0,12 & 5 & 0,31 & 0,0998 & 0,31 & 0,74 \\
\hline Lauraceae & Nectandra megapotamica (Spreng.) Mez & 5 & 0,59 & 20 & 1,24 & 0,1319 & 0,41 & 2,24 \\
\hline Lauraceae & Ocotea lancifolia & 2 & 0,24 & 10 & 0,62 & 0,3904 & 1,20 & 2,06 \\
\hline Lauraceae & Ocotea pulchella Mart. & 2 & 0,24 & 10 & 0,62 & 0,5458 & 1,68 & 2,54 \\
\hline
\end{tabular}

Continua ... 
TABELA 1: Continuação ...

\begin{tabular}{|c|c|c|c|c|c|c|c|c|}
\hline FAMÍLIA & ESPÉCIES & DA & DR & FA & FR & DoA & DoR & $\mathrm{VI}$ \\
\hline Meliaceae & Cedrela fissilis Vell. & 2 & 0,24 & 10 & 0,62 & 0,3595 & 1,11 & 1,97 \\
\hline Mimosaceae & Parapiptadenia rigida (Benth.) Brenan & 11 & 1,30 & 25 & 1,55 & 0,5272 & 1,62 & 4,47 \\
\hline Myrsinaceae & Myrsine umbellata Mart. & 2 & 0,24 & 10 & 0,62 & 0,0427 & 0,13 & 0,99 \\
\hline Myrsinaceae & Myrsine sp. & 1 & 0,12 & 5 & 0,31 & 0,0568 & 0,17 & 0,60 \\
\hline Myrtaceae & Blepharocalyx salicifolius Berg & 39 & 4,60 & 90 & 5,59 & 1,5190 & 4,67 & 14,86 \\
\hline Myrtaceae & Campomanesia xanthocarpa Berg & 30 & 3,54 & 75 & 4,66 & 0,5460 & 1,68 & 9,88 \\
\hline Myrtaceae & Eugenia pluriflora DC. & 3 & 0,35 & 10 & 0,62 & 0,0329 & 0,10 & 1,07 \\
\hline Myrtaceae & Eugenia pyriformis Camb. & 3 & 0,35 & 15 & 0,93 & 0,0351 & 0,11 & 1,39 \\
\hline Myrtaceae & Eugenia uniflora $L$. & 1 & 0,12 & 5 & 0,31 & 0,0154 & 0,05 & 0,48 \\
\hline Myrtaceae & Eugenia uruguayensis $\mathrm{Camb}$. & 1 & 0,12 & 5 & 0,31 & 0,0211 & 0,06 & 0,49 \\
\hline Myrtaceae & $\begin{array}{l}\text { Myrceugenia glaucencens (Camb.)L. et } \\
\text { Kaus. }\end{array}$ & 6 & 0,71 & 20 & 1,24 & 0,0942 & 0,29 & 2,24 \\
\hline Myrtaceae & Myrceugenia miersiana (Gardn.) L. et Kaus. & 3 & 0,35 & 10 & 0,62 & 0,0724 & 0,22 & 1,19 \\
\hline Myrtaceae & Myrceugenia sp. & 1 & 0,12 & 5 & 0,31 & 0,0134 & 0,04 & 0,47 \\
\hline Myrtaceae & Myrcia bombycina (Berg) Legr. & 5 & 0,59 & 20 & 1,24 & 0,1303 & 0,40 & 2,23 \\
\hline Myrtaceae & Myrcia obtecta (Berg) Kiaersk. & 36 & 4,25 & 65 & 4,04 & 0,8035 & 2,49 & 10,78 \\
\hline Myrtaceae & Myrcia selloi (Spreng) Silveira & 2 & 0,24 & 10 & 0,62 & 0,0342 & 0,11 & 0,97 \\
\hline Myrtaceae & Myrcianthes gigantea (Legr.)Legr. & 17 & 2,00 & 50 & 3,11 & 0,8835 & 2,72 & 7,83 \\
\hline Myrtaceae & Myrcianthes pungens (Berg) Legr. & 5 & 0,59 & 20 & 1,24 & 0,2229 & 0,69 & 2,52 \\
\hline Myrtaceae & Myrciaria delicatula (DC.) Berg. & 97 & 11,44 & 100 & 6,21 & 1,6050 & 4,94 & 22,59 \\
\hline Myrtaceae & Myrciaria floribunda Legr. & 3 & 0,35 & 5 & 0,31 & 0,0341 & 0,10 & 0,76 \\
\hline Myrtaceae & Myrrhinium atropurpureum Schott & 15 & 1,77 & 50 & 3,11 & 0,2636 & 0,81 & 5,69 \\
\hline Myrtaceae & Siphoneugenia reitzii Legr. & 3 & 0,35 & 5 & 0,31 & 0,0470 & 0,14 & 0,80 \\
\hline Polygonaceae & Ruprechtia laxiflora Meiss. & 10 & 1,18 & 35 & 2,17 & 0,3282 & 1,06 & 4,41 \\
\hline Proteaceae & Roupala brasiliensis Klotz. & 3 & 0,35 & 10 & 0,62 & 0,0497 & 0,15 & 1,12 \\
\hline Rosaceae & wii Koehne & 7 & 0,83 & 35 & 2,17 & 0,1821 & 0,56 & 3,56 \\
\hline Rosace & Quillaja brasiliensis Mart. & 2 & 0,24 & 5 & 0,31 & 0,0480 & 0,15 & 0,70 \\
\hline Rutaceae & $\begin{array}{l}\text { Zanthoxylum kleinii (R. S. Kowan) } \\
\text { Waterman }\end{array}$ & 67 & 7,90 & 95 & 5,90 & 3,7124 & 11,42 & 25,22 \\
\hline Rutace & Zhanthoxylum rhoifolium $\mathrm{L}$. & 4 & 0,47 & 15 & 0,93 & 0,0498 & 0,15 & 1,55 \\
\hline Sapindaceae & Allophylus edulis (St.Hil.) Radlk. & 5 & 0,59 & 15 & 0,93 & 0,0598 & 0,18 & 1,70 \\
\hline Sapindaceae & Allophylus guaraniticus Camb. & 1 & 0,12 & 5 & 0,31 & 0,0241 & 0,07 & 0,50 \\
\hline Sapindaceae & orualic Comb & 38 & 4,48 & 55 & 3,42 & 0,9473 & 2,92 & 10,82 \\
\hline Sapindaceae & eagnoides Radlk. & 125 & 14,70 & 95 & 5,90 & 4,3313 & 13,33 & 33,93 \\
\hline Simaroubaceae & Picrasma crenata Vell. & 3 & 0,35 & 15 & 0,93 & 0,0400 & 0,12 & 1,40 \\
\hline Styracaceae & Styrax leprosum Hook. et Arn & 4 & 0,47 & 20 & 1,24 & 0,1525 & 0,47 & 2,18 \\
\hline Symplocaceae & Symplocos uniflora (Pohl) Benth. & 4 & 0,47 & 20 & 1,24 & 0,1562 & 0,48 & 2,19 \\
\hline Solanaceae & Solanum intermedium & 1 & 0,12 & 5 & 0,31 & 0,0397 & 0,12 & 0,55 \\
\hline \multirow[t]{2}{*}{ Tiliaceae } & Luehea divaricata Mart. & 2 & 0,24 & 5 & 0,31 & 0,1321 & 0,41 & 0,96 \\
\hline & Mortas & 69 & 8,14 & 100 & 6,21 & 2,4225 & 7,46 & 21,81 \\
\hline 23 & TOTAL & 848 & 100,00 & 1610 & 100 & 32,491 & 100,00 & 300,00 \\
\hline
\end{tabular}

Em que: $\mathrm{DA}=$ densidade absoluta; $\mathrm{DR}=$ densidade relativa; $\mathrm{FA}=$ frequiência absoluta; $\mathrm{FR}=$ freqüência relativa; DoA = dominância absoluta; DoR = dominância relativa; $\mathrm{VI}$ = Valor de importância.

Foram encontradas 848 indivíduos por hectare, distribuídos em 54 espécies e 23 famílias botânicas. A família Myrtaceae com 18 espécies, foi a mais característica e representativa da comunidade analisada, seguida de Lauraceae e Sapindaceae com quatro espécies, Flacourtiaceae com três espécies e Anacardiaceae, Aquifoliaceae, Euphorbiaceae, Myrsinaceae, Rosaceae e 
Rutaceae com duas espécies. Essas dez famílias representam aproximadamente $76 \%$ das espécies encontradas na floresta.

A diversidade de espécies arbóreas, arvoretas e arbustos da família Myrtaceae, é expressiva (18 espécies vegetais presentes no hectare) e concorda com os trabalhos anteriores de RAMBO (1951), KLEIN (1984) e JARENKOW (1985), afirmando que essas comunidades vegetais da Floresta Ombrófila Mista constituem um importante centro de dispersão da família Myrtaceae em que se observa desde árvores de grande portec até arvoretas e arbustos de pequeno porte que habitam o sub-bosque da vegetação.

O Índice de diversidade de Shannon encontrado $\left(\mathrm{H}^{\prime}=3,0\right)$ representa uma diversidade mediana que pode ser esperado para uma floresta com alguma intervenção antrópica, no estádio de sucessão secundária, e no domínio da floresta Ombrófila Mista. Desconsiderando os diferentes procedimentos de amostragem, JARENKOW e BATISTA (1987) encontraram um valor de 2,93 para uma floresta Ombrófila Mista secundária, em Esmeralda - RS e colocam esse valor entre um dos mais baixos já encontrados para florestas com o componente arbóreo pluriestratificado.

As espécies Matayba elaeagnoides (camboatá-branco), Zanthoxylum kleinii (juvevê), Myrciaria delicatula (camboim), Lithraea brasiliensis (bugreiro), Erythrozylum. deciduum (cocão), Araucaria angustifolia (pinheiro-brasileiro), Blepharocalyx salicifolius (murta), Cupania vernalis (camboaté-vermelho) e Myrcia obtecta (guamirim-branco) foram as que apresentaram maiores valores de importância na floresta e por isso, são as mais características da associação analisada (Figura 1). Somam em conjunto 59,6 \% do Valor de Importância total da floresta.

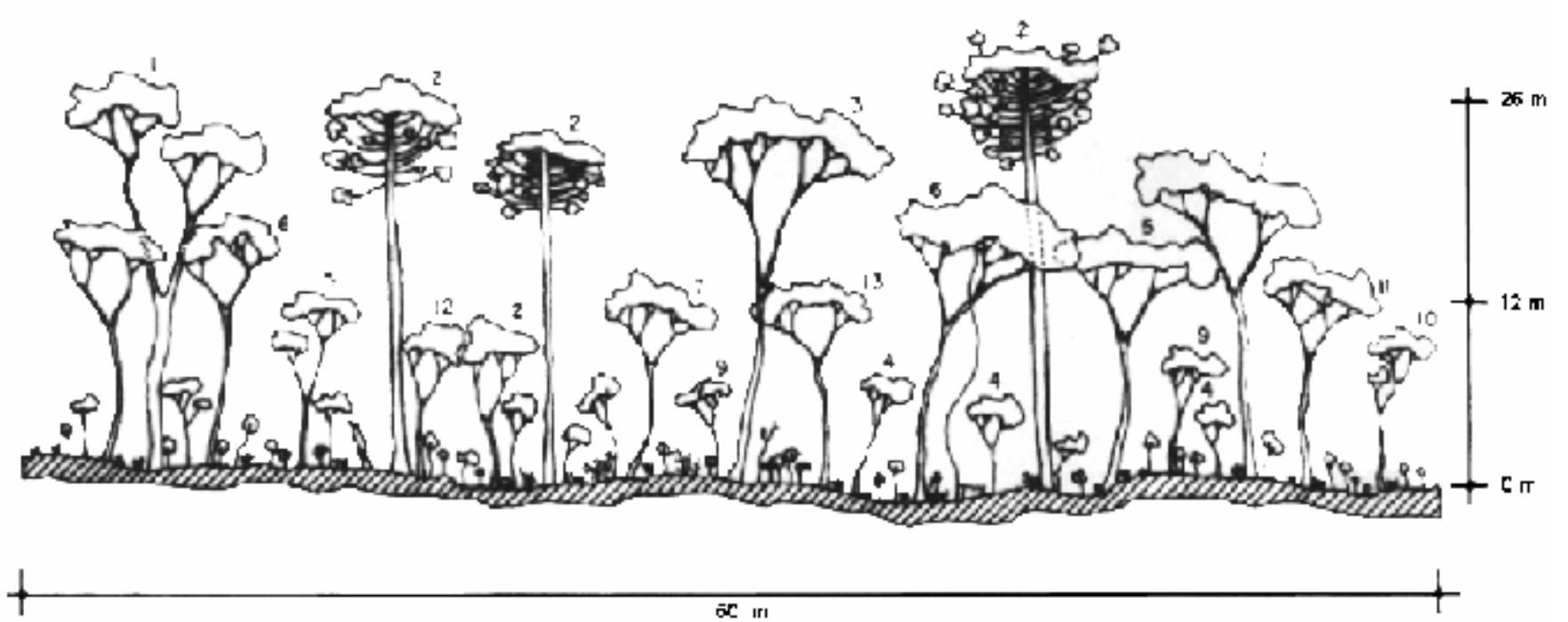

1. Matayba elaeagnoides, 2. Araucaria angustifolia, 3. Zanthoxylum kleinii, 4. Myrciaria delicatula, 5. Erythroxylum deciduum, 6. Blepharocalyx salicifolius, 7. Lithraea brasiliensis, 8. Eugenia pyriformis, 10. Campomanesia xanthocarpa, 11. Sebastiania commersoniana, 12. Myrrhinium atropurpureum, 13. Cupania vernalis.

FIGURA 1: Representação esquemática da estrutura da vegetação no centro da amostra. 
Matayba elaeagnoides (camboatá-branco) e Myrciaria delicatula (camboim), foram as espécies com maior densidade de indivíduos por hectare. Representaram $26,14 \%$ do número de indivíduos total da floresta. Estas, juntamente com Erythrozylum. deciduum (cocão), Zanthoxylum kleinii (juvevê) e Lithraea brasiliensis (bugreiro), somam 48,78\% do total de indivíduos da floresta. Com relação à dominância, apresentaram destaque as espécies: Matayba elaeagnoides (camboatábranco), Araucaria angustifolia (pinheiro-brasileiro), Zanthoxylum kleinii (juvevê) e Lithraea brasiliensis (bugreiro), por apresentarem indivíduos de maiores diâmetros na floresta. Representam $47,76 \%$ da área basal total encontrada. A densidade de pinheiros (23 árvores/hectare), situa-se um pouco abaixo da densidade encontrada em florestas secundárias na região (50-100 árvores/hectare) (BRENA, dados não-publicados). Isto se deve à intensa exploração realizada na floresta há cerca de 20 anos, onde foi extraído grande quantidade de pinheiros de maiores diâmetros.

\section{Padrões de distribuição espacial das espécies}

As estimativas dos padrões de distribuição espacial, das espécies arbóreas amostradas no hectare, podem ser encontradas na Figura 2. Observou-se uma relação decrescente da Razão Variância/Média do número de indivíduos das espécies com DAP $\geq 9,6 \mathrm{~cm}$. As espécies vegetais, com maiores valores de agregação, são também as espécies que possuem maior Média e Variância do número de indivíduos na amostra.

Espécies fortemente agregadas apresentam alta Razão Variância/Média, como as duas espécies de camboim (Syphoneugenia reitzii e Myrciaria delicatula) e guaçatunga-branca (Banara tomentosa). Outra característica importante da distribuição é que a maior parte das espécies vegetais estudadas, apresentaram os valores obtidos da Razão Variância/Média superiores a 1, destacando que as principais espécies da comunidade vegetal apresentam uma distribuição espacial agregada ou, tendendo à agregação na vegetação.

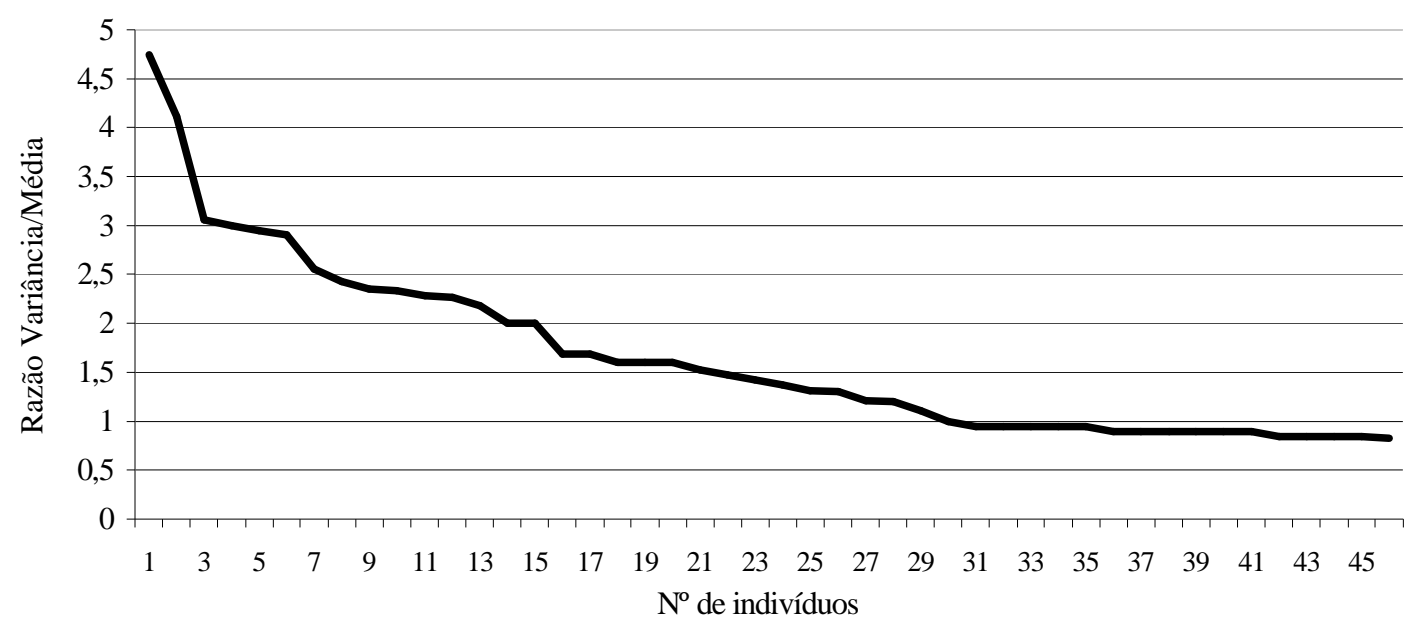

FIGURA 2: Distribuição da Razão Variância/Média para as espécies com DAP $\geq 9,6 \mathrm{~cm}$, Floresta Ombrófila Mista, Nova Prata, RS. 
Por outro lado, as espécies com uma baixa densidade, menos de 2 indivíduo por hectare (35,55\% do total) apresentaram médias inferiores a 1 e, conseqüientemente, uma distribuição espacial aleatória, com menores valores de Razão Variância/Média. Para as espécies vegetais de grande porte como Cedrela fissilis (cedro) e Ruprechtia laxiflora (marmeleiro-do-mato), esse padrão aleatório, com baixa densidade de indivíduos, pode aproximar-se de sua distribuição natural na floresta, por serem espécies de maior longevidade.

Analisando a estrutura espacial da comunidade arbórea estudada, pode-se observar uma grande participação de espécies vegetais com distribuição espacial agregada ou com tendência à agregação (Figura 3). Esse resultado parece refletir a elevada densidade da vegetação (848 ind/hectare) como conseqüência do estágio de renovação que esta se encontra, com uma participação acentuada de árvores e arvoretas de pequeno porte, tendendo a formar pequenas e densas manchas na vegetação.

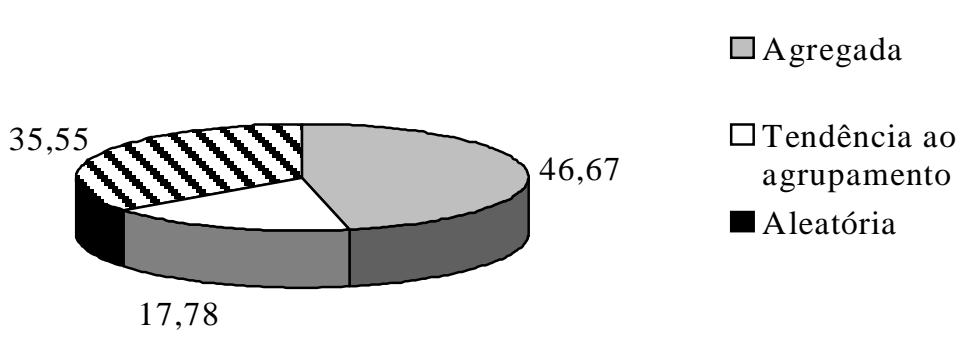

FIGURA 3: Proporção dos diferentes padrões estimados de distribuição espacial para um hectare de Floresta Ombrófila Mista, Nova Prata, RS.

As espécies, com distribuição aleatória (35,5\%), encontram-se bem representadas na vegetação e assumem importância na Floresta Ombrófila Mista estudada. As espécies com somente um indivíduo amostrado não foram incluídas na análise em conjunto, sendo consideradas, contudo, como espécies com distribuição aleatória. Essas espécies vegetais, possivelmente necessitam de uma maior área de amostragem e uma distribuição diferenciada das unidades amostrais, para uma melhor descrição do seu padrão de distribuição de seus indivíduos no espaço.

Na floresta Tropical da Amazônia, CARVALHO (1992) descreve um percentual de 47\% das espécies arbóreas (104 sp.) com distribuição agregada, ocorrendo com baixas densidades, entre um e sete indivíduos $\mathrm{ha}^{-1}$, como Bertholletia excelsa, Lecythis lurida e Manilkara huberi. Do total das espécies estudadas, $38 \%$ foram abundantes, com mais de sete árvores ha $^{-1}$ como Carapa guianensis e Couratari oblongifolia e $15 \%$ foram descritas como espécies raras (aleatórias), com uma média inferior a uma árvore $\mathrm{ha}^{-1}$, por exemplo Aniba guianensis, Caraipa grandiflora, entre outras.

Em formações secundárias, pode ser encontrada uma elevada densidade de árvores por hectare, representados por arvoretas de pequeno porte que habitam os primeiros estratos da vegetação e indivíduos jovens de árvores de grande porte do dossel da floresta. As árvores, com maiores diâmetros e alturas, muitas vezes, são representadas por indivíduos em senescência natural e 
com um maior espaçamento entre os indivíduos, como no caso de Ruprechtia laxiflora (marmeleirodo-mato) e Ocotea lancifolia (canela-pilosa) que apresentam uma baixa densidade na vegetação e uma distribuição aleatória de seus indivíduos adultos na comunidade vegetal estudada.

Entre as espécies com distribuição agregada, foram encontradas duas formas de agregação natural: espécies com uma maior densidade (maior média $=\mu$ ) e maiores valores de Razão Variância/Média (R), como Matayba elaeagnoides (camboatá-branco), Myrciaria delicatula (camboim), Erythroxylum deciduum (cocão) e Zanthoxylum kleinii (juvevê); e espécies agregadas, com baixos valores de Densidade (menores médias) e altos valores de Razão variância/Média (R), como Syphoneugenia reitzii (camboim-graúdo), Sebastiania commersoniana (branquilho), Banara tomentosa (guaçatunga-branca) e Myrciaria floribunda (guamirim). Essas espécies apareceram em poucas unidades na amostra de 1 hectare, ocorrendo no entanto, com vários indivíduos muito próximos entre si, resultando em maiores valores de Variância do número de indivíduos no total das unidades amostrais e um padrão agregado em menor escala.

Também apresentaram distribuição agregada outras espécies importantes da floresta, como Araucaria angustifolia (pinheiro-brasileiro), Cupania vernalis (camboatá-vermelho), Campomanesia xanthocarpa (guabiroba), Luehea divaricata (açoita-cavalo) e Roupala brasiliensis (carvalho-brasileiro), ou com tendência a agregação, como Prunus sellowii (pessegueiro-do-mato), Parapiptadenia rigida (angico-vermelho) e Blepharocalyx salicifolius (murta).

Uma maior participação de espécies formando manchas na vegetação (distribuição espacial agregada), são descritas também para outros tipos de vegetação florestal. Investigando os padrões de distribuição espacial, usando análise espectral bidimensional da área basal, de 64 espécies arbóreas da Floresta tropical em Sarawak- Malásia, NEWBERY et al. (1986) encontraram 30 (46,8 \%) das espécies formando manchas (padrão agregado) na vegetação. Esses resultados são discutidos pelos autores, como uma importante relação entre a matriz de solos do local, a topografia do terreno e a ocorrência de clareiras de duas escalas de tamanho diferentes (0,02 e 0,1-0,2 hectares). Nos sítios mais desenvolvidos da floresta Semidecídua de Misiones - Argentina, as espécies Balfourodendron riedelianum (guatambú), Ocotea puberula (canela-guaicá), Prunus subcoriacea (pessegueiro-bravo) e Rapanea sp. (capororoca) ocorrem abundantemente na vegetação, formando manchas e em forma repetida nas amostras. Outras espécies, como Sebastiania brasiliensis (branquilho-leiteiro) e Cupania vernalis (camboatá-vermelho) encontravam-se distribuídas homogeneamente na superfície amostrada, mas com uma reduzida área basal (CRISTÓBAL et al., 1996).

A estrutura espacial, mesmo em pequena escala, como no presente estudo, destaca os padrões locais de distribuição das espécies vegetais na comunidade. As espécies, com distribuição agregada, ocorreram na amostra em pequenas manchas, formando altos valores de médias e de agregação (Razão V/M). As espécies aleatórias ocorrem mais espalhadas na vegetação, com menores valores das médias no número de indivíduos e baixos valores da Razão (geralmente menores que um) e podem, em determinados casos, apresentarem problemas de regeneração natural. 


\section{CONCLUSÕES}

A amostra de vegetação analisada representa uma floresta primária que sofreu, há cerca de 20 anos, intensa exploração seletiva de indivíduos comerciais de maior porte, apresenta um número mediano de espécies vegetais (54 espécies com DAP $\geq$ a 9,6 cm) e encontra-se em processo de recuperação. As famílias Myrtaceae, Lauraceae, Flacourtiaceae e Rutaceae apresentaram as maiores riquezas em espécies e destacam-se na estrutura e fisionomia do dossel da floresta.

$\mathrm{Na}$ floresta Ombrófila Mista estudada, foi encontrada uma elevada densidade de indivíduos por hectare (848 ind. com DAP $\geq$ a 9,6 cm), formando pequenas manchas ou agregações na amostra de vegetação. Para o total das espécies avaliadas, foi observado um maior número de espécies com distribuição espacial agregada (46,6 \%) ou com tendência à agregação (17,7 \%), e também uma expressão significativa de espécies aleatórias $(35,5 \%)$. O fato da maioria das espécies apresentarem distribuição agregada ou com tendência agregação comprova que a floresta se encontra em fase de renovação, após ter sofrido uma exploração seletiva.

$\mathrm{Na}$ associação vegetal analisada no presente estudo, destacam-se as espécies Matayba elaeagnoides (camboatá-branco)) e Myrciaria delicatula (camboim), por meio do elevado número de indivíduos que apresentam, distribuídas de forma fortemente agregada na amostra. A diversidade de espécies de Myrtaceae é notável no sub-bosque e nos estratos inferiores da floresta e destaca essa comunidade vegetal, como um importante centro de dispersão dessa família.

\section{AGRADECIMENTOS}

À CAPES, pela concessão de bolsa ao primeiro autor. Ao curso de Pós-Graduação em Engenharia Florestal, pelo apoio. Aos botânicos Marcos Sobral e Lílian A. Mentz da UFRGS, pela identificação de material botânico. Ao colega Edson L. Bolfe, pelo auxílio na digitalização do perfil da vegetação. Ao Sr. Vilcêncio Palludo, pela permissão de trabalhar na Fazenda Tupi e aos seus funcionários, pela acolhida. Aos acadêmicos Lucas H. Simm e Rodrigo R. Lima, pelo ajuda na coleta dos dados.

\section{REFERÊNCIAS BIBLIOGRÁFICAS}

BARROS, P.L.C. Estudo Fitossociológico de uma floresta tropical úmida no Planalto de Curuá-una, Amazônia Brasileira. 1986. 147p. Tese (Doutorado em Engenharia Florestal) - Setor de Ciências Agrárias, Universidade Federal do Paraná, Curitiba.

BELEM, M. Structure and dynamics of two gallery forests in the Biosphere Reserve of the Hipopotamous Pool; Burkina Faso, West Africa. In: INTERNATIONAL SIMPOSIUM ON ASSESMENT AND MONITORING OF FORESTS IN TROPICAL DRY REGIONS WITH SPECIAL REFERENCED TO GALLERY FORESTS, 1997, Brasília. Anais... Brasília: UnB, 1997. p.329-337. 
BRENA, D. A. et al. Inventário florestal da Fazenda Tupi Nova Prata, RS. Santa Maria: CEPEF/FATEC/UFSM, 1990. 68 p.

CAIN, S. A.; CASTRO, G. M. O.; PIRES, J. N. et al. Application of some phytosociological techniques to Brazilian rain forests. Amer. J. Bot., New York, v.43, n.3, p.911-941, 1956.

CALDATO, S.L. Dinâmica populacional de Ocotea porosa (Lauraceae) na Floresta Ombrófila Mista em Caçador, SC. 1998, 89 p. Dissertação (Mestrado em Engenharia florestal) - Universidade Federal de Santa Maria, Santa Maria.

CARVAlHO, J.O. P. de. Abundância, frequiência e grau de agregação de Pau-rosa (Aniba duckei) na Floresta Nacional do Tapajós. Belém: Embrapa-CPATU, 1983. 24 p. (Boletim de Pesquisa, 53).

. Structure and dynamics of a logged over Brazilian Amazonian rain forest. 1992. 215 p. Thesis (D. Phil.) - University of Oxford, Oxford.

CONDES, S.; MARTINEZ-MILLAN, J. Comparacion entre los índices de distribuicion espacial de arboles mas usados en el ambito forestal. Invest. Agr.: Sist. Recur. For., v.7, n.1/2, p.173-187, 1998.

CRISTÓBAL, L.L.; GRANCE, L.A; MAIOCCO, D.M. et al. Estructura e composicion floristica del bosque nativo, en el predio guarani. Yvyraretá. Missiones-Argentina, p.30-36, 1996.

DAJÓZ, R. Ecologia geral. São Paulo: Vozes, 1972. 472 p.

DAUBENMIRE, R. Plant communities: a textebook of plant synecology. New York: Harper \& Row, 1968. $300 \mathrm{p}$.

FINOL, H. Nuevos parametros a considerarse en el analisis estructural de las selvas virgenes tropicales. Ver. For. Venez., Mérida, v.14, n.21, p.29-42, 1971.

HOWE, H.F. Scater-and-clump-dispersal and seedling demography: hypothesis and implications. Oecologia, v. 79, p.417-426, 1989.

HOWE, H.F.; WESTLEY, L.C. Ecological relationships of plants and animals. New York: University Press, 1988.

HUSTON, .M.A. Biological Diversity: the coexistence of species on chanching landscapes. Cambridge: Cambrige University Press, 1994.

INOUE, M. T.; RODERJAN, C.V.; KUNIYOSHI, Y.S. Projeto madeira do Paraná. Curitiba: FUPEF/UFPR, 1984. 260 p.

JANKAUSKIS, J. Avaliação de técnicas de manejo florestal. Belém: SUDAM, 1990. 143 p.

JARDIM, F. C. S. Distribuição espacial de espécies arbóreas da Floresta Equatorial de Terra Firme. Acta Amazônica, Manaus, v.19, p.371-382, 1989.

_. A relação entre abundância e frequiência na Floresta Equatorial de Terra Firme. Acta Amazônica, Manaus, v.20, p.185-192, 1990.

JARENKOW, J. A. Composição florística e estrutura da Mata com Araucária na Estação Ecológica de Aracuri, Esmeralda, Rio grande do Sul. 1985. 82 p. Dissertação (Mestrado em Botânica) Universidade Federal do Rio Grande do Sul, Porto Alegre.

JARENKOW, J. A.; BATISTA, L.R.M. Composição florística e estrutura da Mata com Araucária na 
Estação Ecológica de Aracuri, Esmeralda, Rio Grande do Sul. Napaea, n 3, p.9-18, 1987.

KLEIN, R.M. Importância sociológica das mirtáceas nas florestas riograndenses. In: CONGRESSO NACIONAL DE BOTÂNICA, 34., 1984, Manaus. Anais... Manaus: Sociedade Botânica do Brasil, 1984. p.367-375.

LAMPRECHT, H. Ensayo sobre unos metodos para el analisis estructural de los bosques tropicales. Acta Cientifica Venezolana, Mérida, v.13, n.2, p.57-65, 1962.

LEITE, P.F.; KLEIN, R.M. Vegetação. In: IBGE.Geografia do Brasil: Região Sul. Rio de Janeiro, 1990. p.113-150.

LONGHI, S.J. A estrutura de uma floresta natural de Araucaria angustifolia (Bert.) O. Ktze., no sul do Brasil. 1980. 198 p. Dissertação (Mestrado em Engenharia Florestal) - Setor de Ciências Agrárias, Universidade Federal do Paraná, Curitiba.

. Agrupamento e análise fitossociológica em comunidades florestais na sub-bacia hidrográfica do Rio Passo Fundo- RS. 1997. 198 p. Tese (Doutorado em Engenharia Florestal) - Setor de Ciências Agrárias, Universidade Federal do Paraná, Curitiba.

McGHINESS, W.C. The relationships between frequency index and abundace as applied to plant populations in a semi-arid region. Ecology, v.15, p.263-382, 1934.

MARCHIORI, J.N.C. Dendrologia das gimnospermas. Santa Maria: UFSM, 1996. 158 p.

MARTINS, F. R. Estrutura de uma floresta mesófila. Campinas: UNICAMP, 1991. 246 p.

MUELLER-DOMBOIS, D.; ELLENBERG, H. Aims and methods of vegetation ecology. New York: John Wiley \& Sons, 1974. 547 p.

NASCIMENTO, A.R.T. Análise estrutural e padrões de distribuição espacial de uma amostra de floresta Ombrófila Mista. 2000. 90 p. Dissertação (Mestrado em Engenharia Florestal) - Universidade Federal de Santa Maria, Santa Maria.

NASI, R. Analysis of the spatial structure of a rattan population in a mixed dipterocarp forest of Sabah (Malaysia). Acta Oecologica, v.34, n.1, p.73-85, 1993.

NEWBERY, D. Mc. C.; RENSHAW, E.; BRÜNIG, E. F. Spatial pattern of trees in Kerangas forests, Sarawak. Vegetatio, v.65, p.77-89, 1986.

RAMBO, B. O elemento andino pinhal rio-grandense. Anais Botânicos do Herbário Barbosa Rodrigues, v.3, n.3, p.3-39, 1951.

. A fisionomia do Rio Grande do Sul: ensaio de monografia natural. 3. ed. São Paulo, 1956.

SCHIMIDT, H.; TORAL, M.; BURGOS, P. Aspectos de estructura y regeneracion natural para el manejo de los bosques de Araucaria-lenga en el Chile. In: PROBLEMAS FLORESTAIS DO GÊNERO ARAUCARIA / FOREST PROBLEMS OF THE GENUS ARAUCARIA, 1979, Curitiba. Anais... Curitiba: FUPEF, 1980. p.159-166.

SENNA, R.M.; WAECHTER, J.L. Pteridófitas de uma floresta com Araucária. 1- Formas biológicas e padrões de distribuição geográfica. Iheringia, Porto Alegre, n.48, p.41-58, 1997.

SILVA, J.N.M.; LOPES, J.C.A. Distribuição espacial de árvores na Floresta Nacional de Tapajós. Belém: EMBRAPA-CPATU, 1982. 14 p. Documentos, 26. 